\title{
Disturbances of Modulating Molecules (FOXP3, CTLA-4/CD28/B7, and CD40/CD40L) mRNA Expressions in the Orbital Tissue from Patients with Severe Graves' Ophthalmopathy
}

\author{
Przemyslaw Pawlowski, ${ }^{1,2}$ Natalia Wawrusiewicz-Kurylonek, ${ }^{3}$ \\ Anja Eckstein, ${ }^{4}$ Joanna Reszec, ${ }^{1}$ Wlodzimierz Luczynski, ${ }^{5}$ \\ Kristian Johnson, ${ }^{6}$ Adam Kretowski, ${ }^{3}$ Alina Bakunowicz-Lazarczyk, ${ }^{2}$ \\ Maria Gorska, ${ }^{3}$ Jacek Szamatowicz, ${ }^{7}$ Lech Chyczewski, ${ }^{1}$ and Janusz Mysliwiec ${ }^{8}$ \\ ${ }^{1}$ Department of Medical Pathomorphology, Cathedral of Biostructure, Medical University of Biatystok, \\ 13 Waszyngtona Street, 15-269 Białystok, Poland \\ ${ }^{2}$ Department of Pediatric Ophthalmology with Strabismus Treatment Unit, Medical University of Bialystok, 17 Waszyngtona Street, \\ 15-274 Bialystok, Poland \\ ${ }^{3}$ Department of Diabetology, Endocrinology and Internal Medicine, Medical University of Białystok, 24A Skłodowskiej-Curie Street, \\ 15-276 Białystok, Poland \\ ${ }^{4}$ Department of Ophthalmology, Essen University Hospital, University of Duisburg-Essen, \\ Hufeland Straße 55, 45122 Essen, Germany \\ ${ }^{5}$ Department of Pediatrics, Endocrinology and Diabetology with Cardiology Division, Medical University of Bialystok, \\ 17 Waszyngtona Street, 15-274 Białystok, Poland \\ ${ }^{6}$ Bayer HealthCare, 10 Kaiser-Wilhelm-Allee, 51373 Leverkusen, Germany \\ ${ }^{7}$ Department of Gynaecology and Oncologic Gynaecology, Medical University of Bialystok, \\ 24A Skłodowskiej-Curie Street, 15-276 Białystok, Poland \\ ${ }^{8}$ Department of Nuclear Medicine, Medical University of Bialystok, 24A Skłodowskiej-Curie Street, \\ 15-276 Białystok, Poland
}

Correspondence should be addressed to Przemyslaw Pawlowski; przemyslaw.pawlowski@umb.edu.pl

Received 16 May 2014; Revised 13 August 2014; Accepted 18 August 2014

Academic Editor: Andrzej Grzybowski

Copyright (C) 2015 Przemyslaw Pawlowski et al. This is an open access article distributed under the Creative Commons Attribution License, which permits unrestricted use, distribution, and reproduction in any medium, provided the original work is properly cited.

Purpose. To evaluate the relationship between the expression of orbital tissue mRNA for FOXP3, CTLA-4/CD28/CD80/CD86, and CD40/CD40 and the severity of Graves' orbitopathy (GO). Material and Methods. Orbital tissue was obtained from 26 patients with GO, with mild $(n=6)$ or severe GO $(n=20)$, and 7 healthy controls. The expression of mRNA of FOXP3, CTLA4/CD28/CD80/CD86, CD40/CD40L was measured by RT-PCR. TCR and CD3 were evaluated by immunohistochemistry. Results. Higher mRNA for FoxP3 (relative expression: 1.4) and CD40 (1.27) and lower expression of CTLA-4 (0.61) were found in the GO tissues versus controls. In severe GO as compared to mild GO higher mRNA expression for FoxP3 (1.35) and CD40 (1.4) and lower expression for CTLA-4 (0.78), CD28 (0.62), and CD40L (0.56) were found. A positive correlation was found between FOXP3 mRNA and CD3 infiltration $(R=0.796, P=0.0000001)$. Conclusions. The enhanced FOXP3 mRNA expression in GO samples may suggest the dysfunction of FOXP3 cells in the severe GO. The diminished mRNA expression of CTLA-4 in severe GO may indicate inadequate $\mathrm{T}$ regulatory function. The enhanced mRNA expression of CD40 in severe GO and negative correlation to CRP mRNA may suggest their role in the active and inactive GO. 


\section{Introduction}

Graves' orbitopathy (GO) is an organ-specific autoimmune disease with a multifactorial etiology, involving genes and environmental triggers, with smoking as a first example, that cause autoimmune dysregulation [1-3]. It is unknown why only a small subset of patients with Graves' disease (GD) develops GO. Vulnerability to GO manifestations most likely is connected with the highly specialized function of the orbital tissue, a unique fat depot that cushions the globe [4].

Most orbital disorders are inflammatory [1] suggesting that orbital fat may be especially prone to robust inflammatory reactions. Indeed, as compared with fibroblasts from other sites, orbital fibroblasts show exaggerated inflammatory responses to various stimuli $[5,6]$. Orbital fibroblasts express CD40, a costimulatory protein present on the surface of many types of cells, including macrophages, lymphocytes, and thyrocytes. CD4+ T cells expressing the CD40 ligand (CD154) directly activate orbital fibroblasts to proliferate through the formation of CD40-CD154 bridges [7-9].

As with other autoimmune diseases, the initiating event results in loss of tolerance and autoreactivity in the orbit and subsequently leads to mononuclear cell infiltration [10]. Since $\mathrm{T}$ cells infiltrating the retrobulbar tissues are likely to play a key role in the pathogenesis of orbital inflammation Forkhead box P3 (FOXP3), CTLA-4/CD28 and its ligands CD80/CD86 have been considered as candidate genes for GO $[11,12]$. FoxP3 is a crucial regulatory factor for the development and function of regulatory $\mathrm{T}$ cells $\left(\mathrm{T}_{\text {regs }}\right)$, and deficiency of the FOXP3 suppresses the regulatory function of $\mathrm{T}_{\text {regs }}$ [13]. Cytotoxic $\mathrm{T}$ lymphocyte antigen 4 (CTLA-4), a molecule specifically and constitutively expressed on $\mathrm{T}_{\text {regs }}$, plays a key role as a negative regulator of T-cell activation [14]. The CTLA-4 gene was reported to be associated with susceptibility to GO and the G allele at exon 1 CTLA-4(49) A/G polymorphism was correlated with severity of GO $[15$, 16]. In addition, FOXP3 and CD40 genetic association was evaluated both in human and in animal models of GO $[17,18]$.

CD80/CD86 (B7-1, B7-2) counter-receptors for CD28/ CTLA-4, expressed mainly on the antigen-presenting cells (APC), renders, that activated $\mathrm{T}$ cells expressing B7 family molecules could act as APC $[12,19]$. Recently, the polymorphisms of the CD86 gene were suggested as genetic markers, which could be helpful for making the diagnosis and prognosis of GO [12].

The present study was designed to investigate the expression of mRNA of the immune regulatory molecules FOXP3, CTLA-4/CD28/CD80/CD86, and CD40/CD40L in the orbital tissues from patients who underwent orbital decompression due to Graves' orbitopathy to assess their role in the inflammatory process.

\section{Materials and Methods}

2.1. Patients and Controls. Human orbital fat/connective tissue was derived from the orbital tissue bank at the Department of Ophthalmology and University of Essen. It was obtained from 26 patients with GO (25 females and 1 male) who underwent orbital decompression. The mean age of patients at the time of surgery was 44.5 years (range: $36-$ 49). Normal orbital connective tissues were derived from 7 individuals ( 6 females and 1 male) undergoing blepharoplastic surgery with no history of GO or any orbital inflammatory disease. The clinical activity score (CAS) of GO was estimated according to Mourits (Mourits et al., 1997). The severity of the eye disease was estimated using a modified classification of no signs or symptoms; only signs, no symptoms; signs only; proptosis; eye muscle involvement; corneal involvement; and sight visual acuity reduction (NOSPECS) described by Eckstein et al. [20]. The mean CAS was 5.92 (1-10) and the mean NOSPECS was 7.46 (2-13).

The study was approved by the Medical Ethics Committee of the University of Essen, Germany and all study participants gave written informed consent.

2.1.1. Grouping of Patients. The patients were grouped according to the CAS and NOSPECS scores on the follow-up at 11-14 months after onset of GO into those with a mild or severe course of GO. The mild signs of GO were described as CAS $<4$ (almost inactive GO) and NOSPECS $<5$ (mild GO), whilst the severe signs were established as CAS $\geq 4$ (still active disease) and/or NOSPECS $\geq 5$ - "severe GO." The examiner who classified the patients was blind to the thyroid status and TRAb values.

Clinical characteristics of patients are shown in Table 1.

2.2. RNA Extraction and cDNA Synthesis. Total RNA from the tissue was isolated and purified with the use of RNeasy Mini Kit (Qiagen, Valencia, CA, USA), following the manufacturer's protocol. One microgram of total RNA was used to prepare cDNA, and cDNA synthesis was performed using SuperScript First-Strand Synthesis System for RT-PCR (Invitrogen, Carlsbad, CA, USA) according to the manufacturer's instructions in the MJ Research Thermal Cycler (Model PTC200, Watertown, MA, USA).

2.3. RT-PCR and Data Analysis. The levels of transcripts were measured by real-time PCR using human genes QuantiTect Assays and QuantiTect Hs_GAPDH Assay (Qiagen, Valencia, CA, USA) as a normalizer. The following genes were assessed: FOXP3, CD152 (CTLA-4), CD28, CD80 (B7-1), CD86 (B72), CD40, CD154 (CD40L), and CRP (C-reactive protein). Real-time PCR was performed in duplicate in $20 \mu \mathrm{L}$, using the QuantiTect SYBR Green PCR Master Mix (Qiagen) applying the manufacturer's instructions, and conducted in the Chromo4 Real-Time PCR Detector (BIO-RAD, USA). A standard curve construction was generated by a series of four dilutions of cDNA of the control group sample in reaction to the house-keeping gene, GAPDH. On the basis of these curves, the levels of total chosen gene transcripts were calculated after their normalization to GAPDH. The value of CT was determined by the first cycle number at which fluorescence was higher than the set threshold value.

2.4. Evaluation of TCR and CD3 Expression by Immunohistochemical Methods. Following the deparaffinization and rehydration, epitope retrieval was carried out in the EnVision 
TABLE 1: Characteristics of patients.

\begin{tabular}{lccc}
\hline & Severe course of GO & Mild course of GO & Mild + severe GO \\
\hline Number of patients & 20 & 6 & 26 \\
Age & $42.3(36-46)$ & $46.2(42-49)$ & 44.5 years $(36-49)$ \\
Gender & $19 \mathrm{~F} ; 1 \mathrm{M}$ & $6 \mathrm{~F}$ & $25 \mathrm{~F} ; 1 \mathrm{M}$ \\
Smokers & $88 \%$ & $50 \%$ & $77 \%$ \\
TRAb (mean \pm SD) & $23 \pm 11.57$ & $2.56 \pm 1.3$ & $18.89 \pm 13.59$ \\
CAS & $7.1(4-10)$ & $2(1-3)$ & $5.92(1-10)$ \\
NOSPECS & $8.55(5-13)$ & $3.83(2-5)$ & $7.46(2-13)$ \\
Proptosis right & $18(14.5-25)$ & $16.25(14-18)$ & $17.5(14-25)$ \\
Proptosis left & $19.5(15-25)$ & $16.5(14-21.5)$ & $17.5(14-25)$ \\
\hline
\end{tabular}

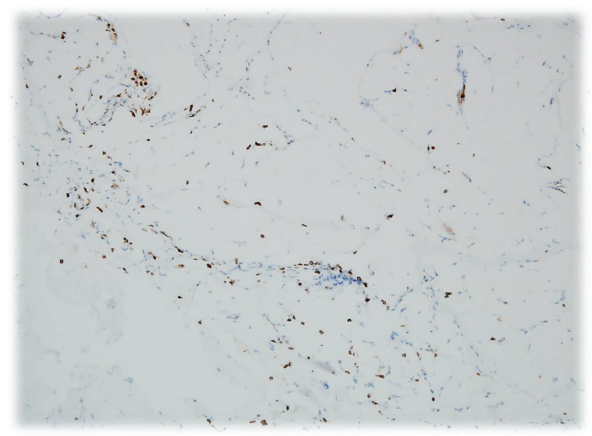

(a)

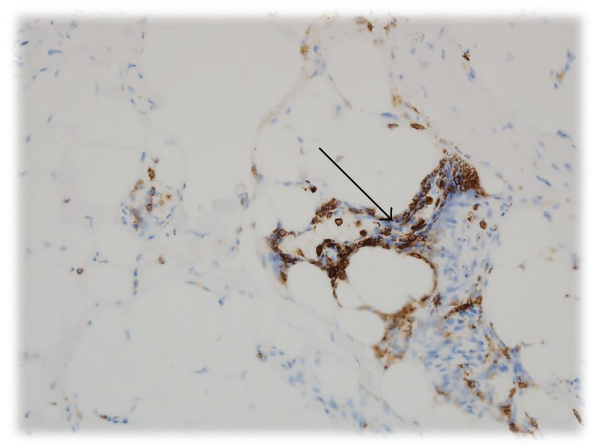

(c)

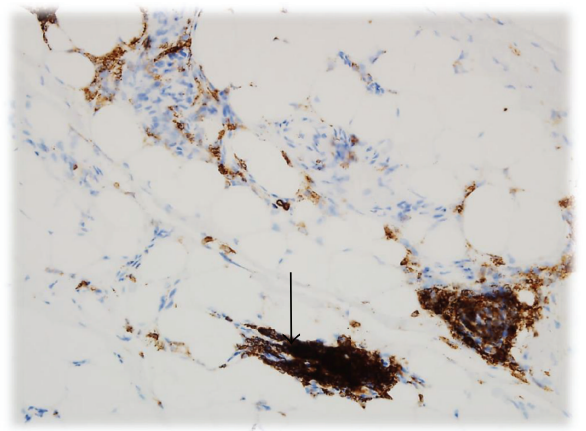

(b)

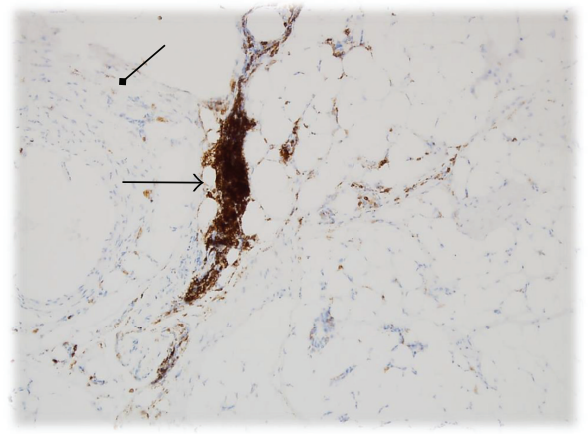

(d)

FIGURE 1: (a) Strong and diffuse TCR expression in lymphoid cells within the mild Graves ophthalmopathy. Magnification: 100x. (b) Focuses of the lymphoid cells aggregation (TCR expression) in the mild Graves ophthalmopathy. Magnification: 200x. ((c), (d)) Strong and diffuse TCR receptor expression in lymphocytes within severe Graves ophthalmopathy. Magnification: 200x, 100x (the arrows show T cells and fibroblasts).

Flex Target Retrieval Solution (DAKO) in high pH. Endogenous peroxidases were blocked by incubating the sections in methanol and 3\% hydrogen peroxidase for 20 minutes. Next slides were incubated with mouse monoclonal antibody against TCR receptor (Novocasta) TCR alpha/beta Antibody (R73) in 1:100 dilution for 30 minutes in room temperature. Against CD3 receptor, were used rabbit polyclonal antibody (Anti-CD3 antibody (ab5690)) in 1:75 dilution for $30 \mathrm{~min}$ utes in room temperature. Visualization reagent EnVision (DAKO) was applied for 30 minutes followed by DAB solution for 10 minutes. The slides were then counterstained with hematoxylin and examined under the light microscope. The intensity of immunostaining was evaluated in random
10 fields under 20x magnification. The results were expressed as the percentage of cells with a strong positive staining as follows: $\leq 10 \%$ positive cells: negative $(-)$, between $11 \%$ and $50 \%(+)$, and $>50 \%$ positive cells $(++)$ (see Table 2 ).

\section{Statistical Analysis}

In order to calculate mRNA expression, a comparative CT method was used for relative quantification, that is, $2^{-\Delta \Delta C T}$, following Livak and Schmittgen [21]. The results were analyzed in Statistica 9.0 for Windows (StatSoft, Poland). Owing to asymmetric data distribution, nonparametric tests 
TABLE 2: Immunohistochemical evaluation grading score for TCR and CD3 of each specimen.

\begin{tabular}{|c|c|c|c|}
\hline Number & Group & TCR & $\mathrm{CD} 3$ \\
\hline 1 & Control & 0 & 0 \\
\hline 2 & Control & 0 & 0 \\
\hline 3 & Control & 0 & 0 \\
\hline 4 & Control & 0 & 0 \\
\hline 5 & Control & 0 & 0 \\
\hline 6 & Control & 0 & 0 \\
\hline 7 & Control & 0 & 0 \\
\hline 8 & Mild GO & 0 & 0 \\
\hline 9 & Mild GO & 1 & 1 \\
\hline 10 & Mild GO & 1 & 1 \\
\hline 11 & Mild GO & 1 & 1 \\
\hline 12 & Mild GO & 1 & 1 \\
\hline 13 & Mild GO & 0 & 0 \\
\hline 14 & Severe GO & 1 & 1 \\
\hline 15 & Severe GO & 1 & 1 \\
\hline 16 & Severe GO & 1 & 1 \\
\hline 17 & Severe GO & 1 & 1 \\
\hline 18 & Severe GO & 2 & 1 \\
\hline 19 & Severe GO & 1 & 1 \\
\hline 20 & Severe GO & 1 & 1 \\
\hline 21 & Severe GO & 2 & 1 \\
\hline 22 & Severe GO & 2 & 1 \\
\hline 23 & Severe GO & 2 & 2 \\
\hline 24 & Severe GO & 1 & 1 \\
\hline 25 & Severe GO & 2 & 1 \\
\hline 26 & Severe GO & 2 & 1 \\
\hline 27 & Severe GO & 1 & 1 \\
\hline 28 & Severe GO & 1 & 1 \\
\hline 29 & Severe GO & 1 & 1 \\
\hline 30 & Severe GO & 1 & 1 \\
\hline 31 & Severe GO & 1 & 1 \\
\hline 32 & Severe GO & 2 & 1 \\
\hline 33 & Severe GO & 1 & 1 \\
\hline
\end{tabular}

Legend for Table 2: Immunohistochemistry scores are as follows: 0 : less than $10 \%$ positive in 10 representative high power fields (HPF), 1: $11 \%-50 \%$ positive cells in $10 \mathrm{HPF}$,

2: more than $50 \%$ positive cells in $10 \mathrm{HPF}$.

Immunohistochemistry was done using DAB chromogen (brown staining).

were used. Significance levels were calculated in accordance with Mann-Whitney $U$ test (differences between the control and examined group). The correlations between the clinical parameters and RT-PCR results were assessed with Spearman's rank correlation test. The level of $P<0.05$ was regarded as significant. Due to the small amount of samples, the relative values (a comparison between the control and examined samples) below 0.75 were regarded as a decreased gene expression, between 0.75 and 1 as a comparable expression, and above 1 as an increased expression [22]. The data are presented as the relative expression of genes in examined samples compared to control samples with the example of individual data in the case of FoxP3 gene. The graphs were prepared in GraphPad Prism 5.0.

\section{Results}

\subsection{CD3 and TCR Expression in Lymphoid Cells In Situ Immunohistochemical Evaluation}

4.1.1. TCR Expression in Mild GO and Severe GO. Focal lymphoid cells aggregation with TCR expression in the mild Graves ophthalmopathy was seen in (4/6 samples) 66.66 $\%$ with mild GO and similarly in $65 \%$ with severe GO (13/20 samples). However, in 7/10 (35\%) with severe GO, the expression of TCR in the lymphoid cell was evaluated as $(++)$ (Figures 1 and 5).

In addition, no expression of CD3 or TCR was revealed in the control tissue specimens (Table 3).

4.1.2. CD3 Expression in Mild GO and Severe GO. $66.66 \%$ with mild GO (4/6) and 95\% with severe GO (19/20) had focal CD3 lymphoid cells infiltrations in the orbital tissue. In addition, in one patient with severe GO (5\%), the infiltration was strong and diffused (Figures 1, 2, 3, 4, and 5 and Table 3)

4.2. The mRNA Expression in GO Tissues and Control Samples. The mRNA for FoxP3, CRP, CD40, and CD86 were present in all the samples $(N=33,100.0 \%)$. The mRNA for CTLA4 was detected in $3 / 7$, that is, $42.8 \%$ of controls, and $19 / 26$, that is, $73.0 \%$ of GO tissue samples. The mRNA for CD28 molecule was noted in $2 / 7$, that is, $28.5 \%$ of controls, and $10 / 26$, that is, $38.4 \%$ of GO tissue samples. The mRNA for CD40L molecule was present only in $1 / 7$, that is, $14.2 \%$ of controls, and $8 / 26$, that is, $30.7 \%$ of GO tissue samples. The mRNA for CD80 was not detected in controls $(0 / 7)$ and only in $8 / 26$, that is, $30.7 \%$, of GO tissue samples. No statistically significant differences were noted between control and examined samples concerning the expression of assessed genes. However, according to our further analysis based on quantitative RT-PCR validation of the mRNA expression changes (as stated in statistical analysis), higher amounts of mRNA for FoxP3 (relative expression: 1.4), CD28 (1.06), CD40 (1.27), CD40L (1.17), and CD86 (1.17) and lower expression of CTLA-4 (0.61) and CRP (0.62) were found in GO as compared to control tissues (see Figure 3).

Further, the expression of mRNA between tissues from patients with severe to mild GO was compared. No significant differences were noted in accordance with Mann-Whitney $U$ test. However, in the analysis of relative amounts of mRNA, we found higher expression of mRNA for FoxP3 (relative expression: 1.35) and CD40 (1.4) and lower expression of mRNA for CTLA-4 (0.78), CD28 (0.62), and CD40L (0.56) in severe GO as compared to mild GO tissue samples. The relative expression of mRNA for assessed molecules is presented in Figure 4.

We found no correlation between the clinical data and results from RT-PCR. However, CRP mRNA correlated 


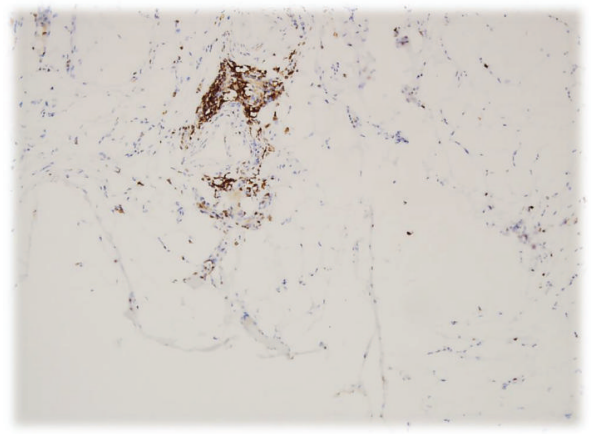

(a)

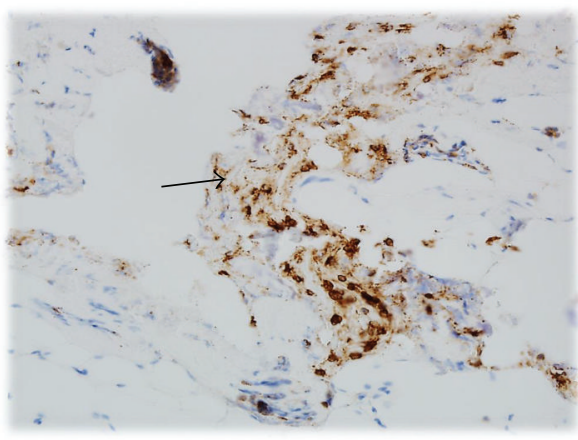

(c)

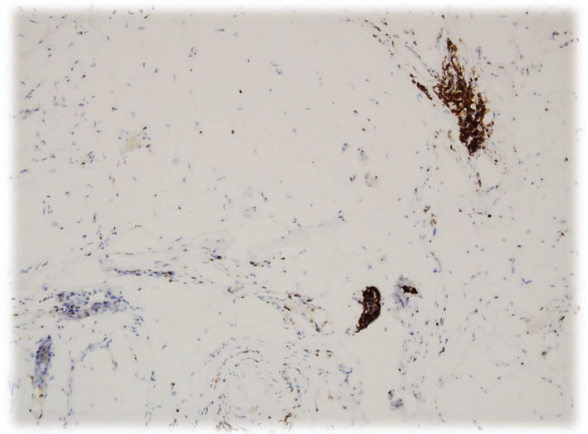

(b)

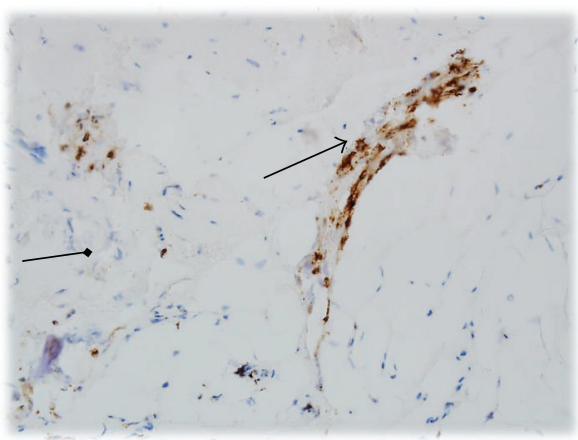

(d)

Figure 2: ((a), (b)) CD3 expression in T cells within the mild Graves ophthalmopathy. Magnification: 100x. ((c), (d)) CD3 strong expression in T cells within the severe Graves ophthalmopathy. Magnification: 200x (the arrows show T cells and fibroblasts).

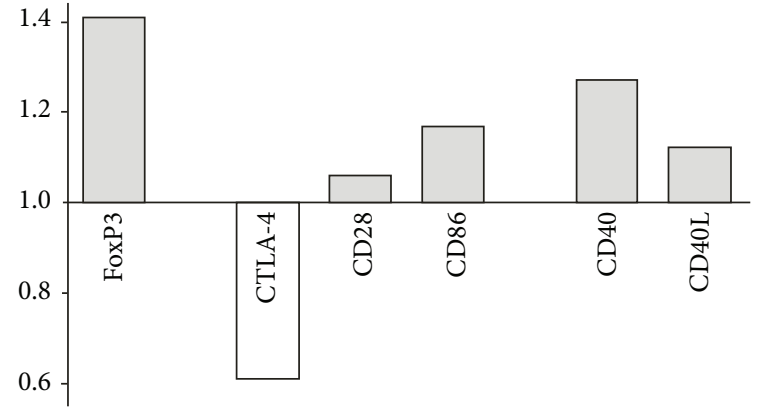

(a)

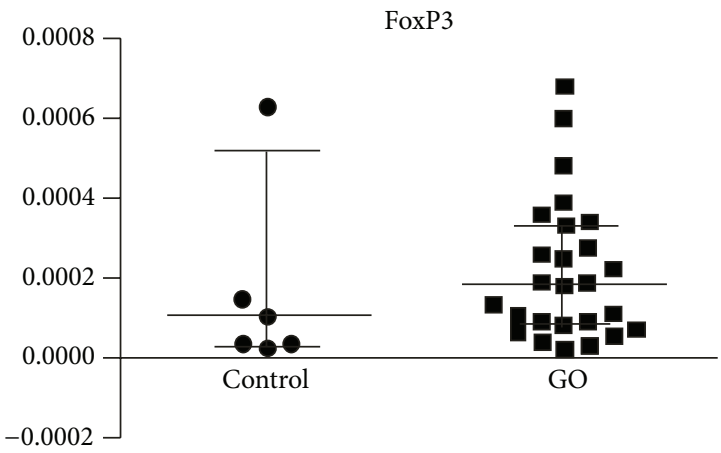

(b)

FIgURE 3: (a) Quantitative RT-PCR validation of the mRNA expression changes for the CD86/CD28/CTLA-4: FOXP3 and CD40/CD40L molecules in GO (mild + severe) orbital tissues compared to control tissue samples. The values above "1.0" on the $y$-axis show the relative higher expression in GO versus control samples and values below "1.0" indicate relatively lower expression. (b) The individual data from RT-PCR displaying the expression of mRNA for transcription factor FoxP3 in control and examined (GO) samples (medians and 25th-75th percentiles $)(P<0.05)$.

positively with FOXP3 $(R=0.520$ and $P=0.03)$ and negatively with CD40 values $(R=-0.757$ and $P=0.000001)$. In addition, a positive correlation was found between FOXP3 mRNA and CD3 infiltration $(R=0.358, P=0.05)$ and a strong correlation was found between TCR and CD3 expression $(R=0.796, P=0.0000001)$ (see Table 4$)$.

\section{Discussion}

The explanation why the connective tissue of the orbit and skin should be singled out for activation in GD concerns possible intrinsic differences in the residential orbital and leg cells as setting up these regions for disease involvement in GD [4]. 


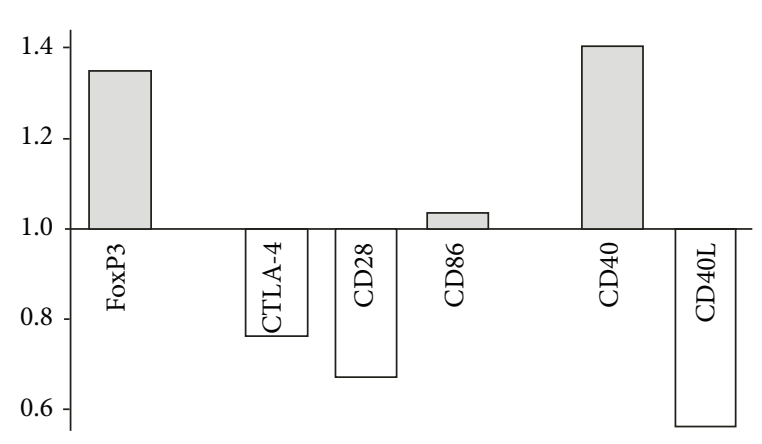

(a)
FoxP3

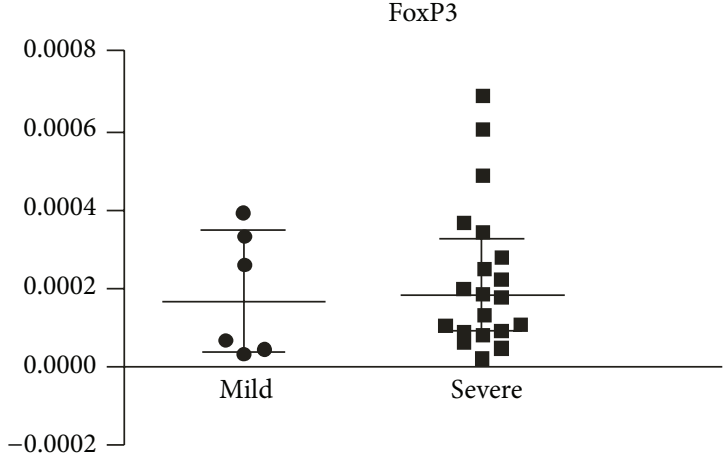

(b)

FIGURE 4: (a) Quantitative RT-PCR validation of the mRNA expression changes for assessed molecules in the severe GO compared to mild GO samples. The values above "1.0" on the $y$-axis show the relatively higher expression in the severe versus mild GO samples and values below "1.0" indicate relatively lower expression. (b) The individual data from RT-PCR displaying the expression of mRNA for transcription factor FoxP3 in severe GO and mild GO samples (medians and 25th-75th percentiles).

TABLE 3: Number of patients and the percentage of (a) TCR and (b) CD3 expression within scores 0,1 , and 2 in examined groups (see the footer of Table 2).

(a)

\begin{tabular}{lcccc}
\hline TCR expression & Control & Mild GO & Severe GO & Sum \\
\hline$\leq 10 \%$ & 7 & 2 & 0 & 9 \\
{$[\%]$} & $100.0 \%$ & $33.34 \%$ & $0.00 \%$ & \\
{$[10 \%-50 \%]$} & 0 & 4 & 13 & 17 \\
{$[\%]$} & $0.00 \%$ & $66.66 \%$ & $65.00 \%$ & \\
$>50 \%$ & 0 & 0 & 7 & 7 \\
{$[\%]$} & $0.00 \%$ & $0.00 \%$ & $35.00 \%$ & \\
\hline
\end{tabular}

(b)

\begin{tabular}{lcccc}
\hline CD3 expression & Control & Mild GO & Severe GO & Sum \\
\hline$\leq 10 \%$ & 7 & 2 & 0 & 9 \\
{$[\%]$} & $100.0 \%$ & $33.34 \%$ & $0 \%$ & \\
{$[10 \%-50 \%]$} & 0 & 4 & 19 & 24 \\
{$[\%]$} & $0.0 \%$ & $66.66 \%$ & $95 \%$ & \\
$>50 \%$ & 0 & 0 & 1 & 1 \\
{$[\%]$} & $0.00 \%$ & $0.00 \%$ & $5 \%$ & \\
\hline
\end{tabular}

TABLE 4: Spearman's correlation table with number of samples, $R$, and $P$ value.

\begin{tabular}{lccc}
\hline & $N$ & $R$ & $P$ value \\
\hline TCR expression \& CD3 expression & 32 & 0.796 & $\mathbf{0 . 0 0 0 0 0 0 1}$ \\
CD3 expression \& CTLA-4 & 20 & -0.375 & 0.1 \\
CD3 expression \& Fox & $\mathbf{3 2}$ & $\mathbf{0 . 3 5 8}$ & $\mathbf{0 . 0 5}$ \\
CD3 expression \& CD40 & 32 & -0.124 & 0.5 \\
CD3 expression \& CD86 & 32 & 0.186 & 0.3 \\
CRP \& Fox & $\mathbf{3 2}$ & $\mathbf{0 . 5 2 0}$ & $\mathbf{0 . 0 0 3}$ \\
CRP \& CD40 & $\mathbf{3 2}$ & $-\mathbf{0 . 7 5 7}$ & $\mathbf{0 . 0 0 0 0 0 1}$ \\
\hline
\end{tabular}

Among other immunoregulatory molecules, CTLA-4 and CD40 in GD and GO have been evaluated most extensively
$[15,23]$. In an early study, Vaidya et al. demonstrated that the G-carrying genotypes of the CTLA4A/G polymorphism are associated with an increase in risk of $\mathrm{GO}$ and are independent of male sex, smoking, and previous radioiodine administration [24]. Since then, many studies have evaluated the CTLA-4 polymorphism in different ethnic groups and its association with ophthalmopathy and Graves' disease [25]. For CTLA-4 function SNP C-318 T, SNP A49G (alanine/threonine polymorphism) and microsatellite- sequence in $3^{\prime}$ UTR was determined [26]. Most recently, Borodic et al. described a case report on treatment with ipilimumab, the monoclonal antibody blocking CTLA-4 mediated T cell suppression, that has led to GO-like syndrome [27]. The decreased expression of the CTLA- 4 mRNA and increased expression of its counter-receptor B7-2 (CD86) in the orbital tissue from GO may explain that patients with GD develop severe GO due to the increased autoimmune reactivity in the orbital venue. Saverino et al. found elevated soluble form of CTLA-4 (sCTLA-4) in serum from patients with autoimmune thyroid disease suggesting a possible role in immune response dysregulation [28]. Thus, the systemic elevation may be caused by increased cleavage of the surface CTLA- 4 and lack of its expression in inflammation site in the orbit.

The increased CD86 mRNA expression found in GO orbital samples may suggest that CD86 costimulation promotes a direct immune response toward Th2 lymphocytes development in the orbital tissue [29]. Th2 differentiation has been shown to be involved in the humoral immune response involving B cells and antibodies production. Sigal et al. found that the CD86 costimulation was more critical for cytotoxic T lymphocytes generation [30].

Similar to CD86, CD40 and CD40L were identified as important B cell activation factors [31]. Most recently, both CD40 and CD86 were found to be elevated in the thyroid of the animal model of GD [32]. In agreement with that, we found an increased level of CD40 mRNA in the orbital tissue samples. In addition, a single nucleotide polymorphism (SNP) $\mathrm{C} / \mathrm{T}$ in the untranslated region of the CD40 gene has been associated with susceptibility to Graves' disease 

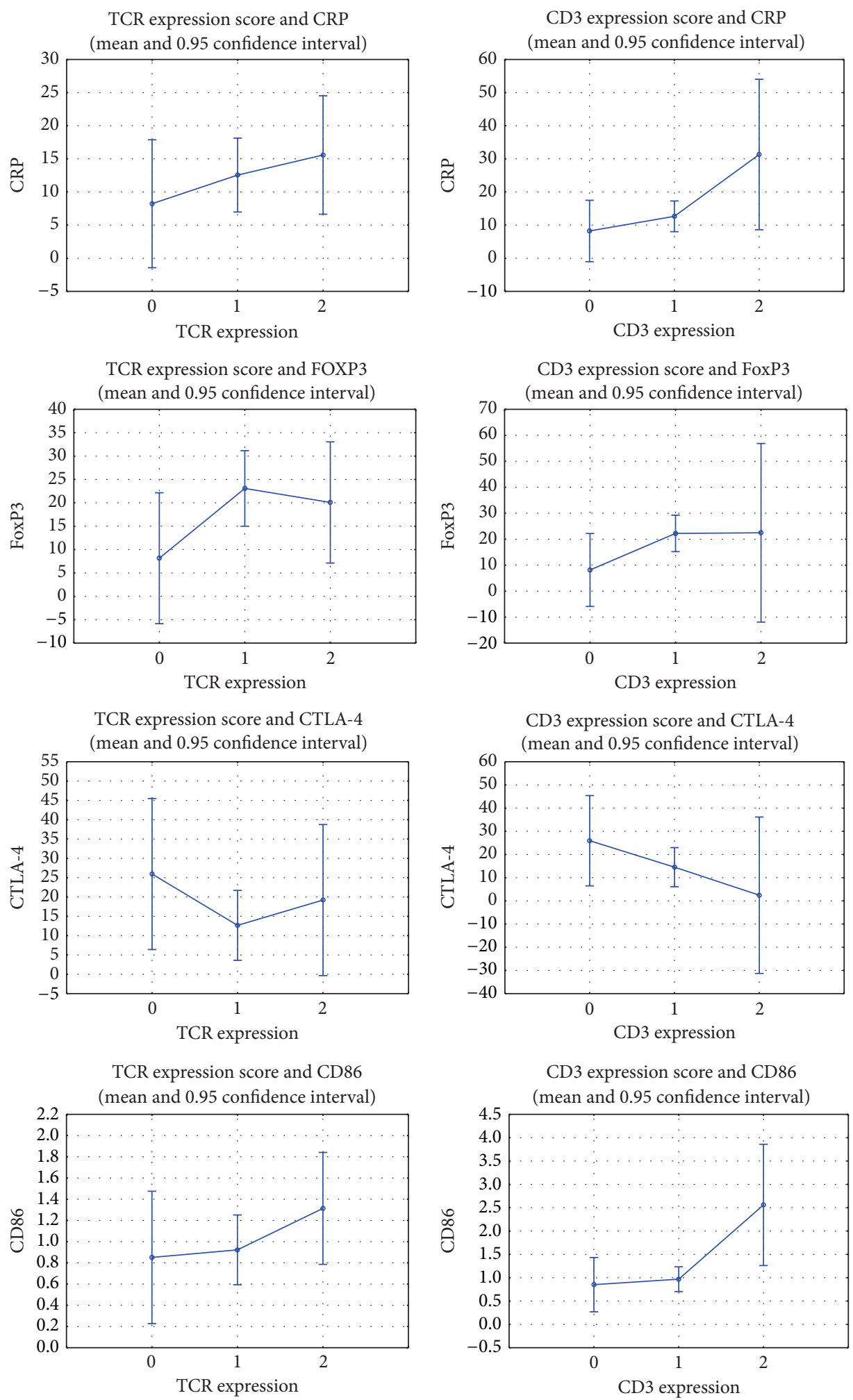

FIGURE 5: TCR and CD3 immunohistochemical grading score groups' ( $0: \leq 10 \%$ positive cells; $1: 11 \%-50 \%, 2:>50 \%$ cells $)$ mean and confidence values of CRP, FOXP3, CTLA-4, and CD86 mRNA. 
in Caucasian [33] and Korean populations [2]. This SNP may alter the translational efficiency of CD40 protein [34]. Jacobson et al. showed that the T-allele makes 15.5\% less CD40 than the C-allele, demonstrating that the effect of the single-nucleotide polymorphism (SNP) on CD40 expression is at the level of translation [34]. Human B cells harboring this SNP expressed $39 \%$ and $27 \%$ higher levels of surface CD40 at rest and activation, respectively, compared with controls. Moreover, this SNP has been linked with the increased production of autoantibodies [35]. However, no difference was detected in steady state CD40 mRNA expression in GD [34].

It is possible that the elevated levels of surface CD40 might amplify cell activation during tissue injury, potentially predisposing the host to GD and perhaps to GO [36]. Since ligation of CD40L on T cells by CD40 B cells or other APC was shown to be necessary for efficient activation of $\mathrm{T}$ cell effector functions, elevated serum concentrations of serum CD40 and CD154 could suggest the systemic activation and explain the increased CD40/CD40L mRNA level in the orbital tissue as a pathway leading to inflammatory infiltration in GO [37].

Direct evidence that any particular candidate antigen or orbital cell type is an autoimmune target in GO has been difficult to obtain due to the lack of available human orbital tissue. However, the loss of tolerance could anyway be the trigger of GO. In agreement with that, we found that the level of FOXP3 mRNA was elevated in GO patients comparing to controls. In addition, the expression of FOXP3 mRNA was also higher in patients with the severe course of GO than in patients with the mild course of GO. These disturbances could be elucidated by the fact that, by preventing the activation of autoreactive pathogenic cells, $\mathrm{CD} 4{ }^{+} \mathrm{CD} 25^{+} \mathrm{FOXP}^{+}$regulatory $\mathrm{T}$ lymphocytes $\left(\mathrm{T}_{\text {regs }}\right)$ have a critical role in the maintenance of self-tolerance and thus in the prevention of autoimmune disease [13]. Hence, the autoimmune response might be more enhanced in GO especially in patients with severe GO. It has been shown that the incubation with polyclonal rabbit anti-T lymphocyte (rATG) globulin increased the frequency of PBMCs of GO patients expressing $\mathrm{T}_{\text {regs }}$-markers (CD25, FOXP3). Kahaly et al. have found that FOXP3/CD4 rATG-induced $\mathrm{T}_{\text {regs }}$ marker was more intensively expressed on GO peripheral blood leucocytes (PBLs) than on GD or normal PBLs [38]. Interestingly, FOXP3 mRNA from peripheral blood was equal in patients with active GD to that in controls [39]. It is possible that in our study the enhanced FOXP3 mRNA could be linked with a polymorphism or a dysfunction of FOXP3 that attempted to compensate the immunological response in its overexpression [18].

\section{Conclusions}

The enhanced FOXP3 mRNA expression in GO samples and its correlation with CD3 and CRP may suggest the involvement and perhaps dysfunction of FOXP3 lymphoid cells in the pathogenesis of severe GO. The diminished mRNA expression of CTLA-4 in severe GO may indicate inadequate $\mathrm{T}$ regulatory function of this molecule in severe course of GO. The enhanced mRNA expression of CD40 in severe versus mild GO and negative correlation to CRP mRNA may suggest their role not only in active but also in the late inactive phase of GO.

\section{Conflict of Interests}

The authors declare that there is no conflict of interests regarding the publication of this paper.

\section{Acknowledgment}

The study was financed by Grants nos. 114-69895 L and 12369676 L of Medical Academy of Bialystok.

\section{References}

[1] R. S. Bahn, “Graves' ophthalmopathy," New England Journal of Medicine, vol. 362, no. 8, pp. 726-774, 2010.

[2] N. Kim and M. P. Hatton, "The role of genetics in Graves' disease and thyroid orbitopathy," Seminars in Ophthalmology, vol. 23, no. 1, pp. 67-72, 2008.

[3] N. Xia, S. Zhou, Y. Liang et al., " $\mathrm{CD} 4^{+} \mathrm{T}$ cells and the Th1/Th2 imbalance are implicated in the pathogenesis of Graves' ophthalmopathy," International Journal of Molecular Medicine, vol. 17, no. 5, pp. 911-916, 2006.

[4] B. S. Prabhakar, R. S. Bahn, and T. J. Smith, "Current perspective on the pathogenesis of Graves' disease and ophthalmopathy," Endocrine Reviews, vol. 24, no. 6, pp. 802-835, 2003.

[5] T. J. Smith, C. C. Tsai, M.-J. Shih et al., "Unique attributes of orbital fibroblasts and global alterations in IGF-1 receptor signaling could explain thyroid-associated ophthalmopathy," Thyroid, vol. 18, no. 9, pp. 983-988, 2008.

[6] M. Meyer Zu Hörste, E. Ströher, U. Berchner-Pfannschmidt et al., "A novel mechanism involved in the pathogenesis of graves ophthalmopathy (GO): clathrin is a possible targeting molecule for inhibiting local immune response in the orbit," Journal of Clinical Endocrinology and Metabolism, vol. 96, no. 11, pp. E1727-E1736, 2011.

[7] C. J. Hwang, N. Afifiyan, D. Sand et al., "Orbital fibroblasts from patients with thyroidassociated ophthalmopathy overexpress cd40: CD154 hyperinduces IL-6, IL-8, and MCP-1," Investigative Ophthalmology and Visual Science, vol. 50, no. 5, pp. 2262-2268, 2009.

[8] S. E. Feldon, D. J. J. Park, C. W. O’Loughlin et al., "Autologous T-lymphocytes stimulate proliferation of orbital fibroblasts derived from patients with Graves' ophthalmopathy," Investigative Ophthalmology and Visual Science, vol. 46, no. 11, pp. 39133921, 2005.

[9] G. D. Sempowski, J. Rozenblit, T. J. Smith, and R. P. Phipps, "Human orbital fibroblasts are activated through CD40 to induce proinflammatory cytokine production," The American Journal of Physiology, vol. 274, no. 3, pp. C707-C714, 1998.

[10] A. K. Eckstein, K. T. Johnson, M. Thanos, J. Esser, and M. Ludgate, "Current insights into the pathogenesis of Graves' orbitopathy," Hormone and Metabolic Research, vol. 41, no. 6, pp. 456-464, 2009.

[11] T. Bednarczuk, B. Gopinath, R. Ploski, and J. R. Wall, "Susceptibility genes in Graves' ophthalmopathy: searching for a needle in a haystack?" Clinical Endocrinology, vol. 67, no. 1, pp. 3-19, 2007. 
[12] W.-L. Liao, R.-H. Chen, H.-J. Lin et al., "The association between polymorphisms of B7 molecules (CD80 and CD86) and graves' ophthalmopathy in a Taiwanese population," Ophthalmology, vol. 118, no. 3, pp. 553-557, 2011.

[13] N. Inoue, M. Watanabe, M. Morita et al., "Association of functional polymorphisms related to the transcriptional level of FOXP3 with prognosis of autoimmune thyroid diseases," Clinical and Experimental Immunology, vol. 162, no. 3, pp. 402406, 2010.

[14] J. Bayry, "Autoimmunity: CTLA-4: a key protein in autoimmunity," Nature Reviews Rheumatology, vol. 5, no. 5, pp. 244-245, 2009.

[15] Y. Ban, T. F. Davies, D. A. Greenberg et al., "Analysis of the CTLA-4, CD28, and inducible costimulator (ICOS) genes in autoimmune thyroid disease," Genes and Immunity, vol. 4, no. 8, pp. 586-593, 2003.

[16] B. Vaidya, E. J. C. Oakes, H. Imrie et al., "CTLA4 gene and Graves' disease: association of Graves' disease with the CTLA4 exon 1 and intron 1 polymorphisms, but not with the promoter polymorphism," Clinical Endocrinology, vol. 58, no. 6, pp. 732735, 2003.

[17] E. M. Jacobson and Y. Tomer, "The CD40, CTLA-4, thyroglobulin, TSH receptor, and PTPN22 gene quintet and its contribution to thyroid autoimmunity: back to the future," Journal of Autoimmunity, vol. 28, no. 2-3, pp. 85-98, 2007.

[18] C. J. Owen, J. A. Eden, C. E. Jennings, V. Wilson, T. D. Cheetham, and S. H. S. Pearce, "Genetic association studies of the FOXP3 gene in Graves' disease and autoimmune Addison's disease in the United Kingdom population," Journal of Molecular Endocrinology, vol. 37, no. 1, pp. 97-104, 2006.

[19] Z.-W. Sun, Y.-H. Qiu, Y.-J. Shi et al., “Time courses of B7 family molecules expressed on activated T-cells and their biological significance," Cellular Immunology, vol. 236, no. 1-2, pp. 146-153, 2005.

[20] A. K. Eckstein, H. Lax, C. Lösch et al., "Patients with severe Graves'ophthalmopathy have a higher risk of relapsing hyperthyroidism and are unlikely to remain in remission," Clinical Endocrinology, vol. 67, no. 4, pp. 607-612, 2007.

[21] K. J. Livak and T. D. Schmittgen, "Analysis of relative gene expression data using real-time quantitative PCR and the 2$\Delta \Delta$ CT method," Methods, vol. 25, no. 4, pp. 402-408, 2001.

[22] S. Glisic, S. Ehlenbach, P. Jailwala, J. Waukau, S. Jana, and S. Ghosh, "Inducible regulatory T cells (iTregs) from recent-onset type 1 diabetes subjects show increased in vitro suppression and higher ITCH levels compared with controls," Cell and Tissue Research, vol. 339, no. 3, pp. 585-595, 2010.

[23] Y. Ban and Y. Tomer, "The contribution of immune regulatory and thyroid specific genes to the etiology of Graves' and Hashimoto's diseases," Autoimmunity, vol. 36, no. 6-7, pp. 367379, 2003.

[24] B. Vaidya, H. Imrie, P. Perros et al., "Cytotoxic T lymphocyte antigen-4 (CTLA-4) gene polymorphism confers susceptibility to thyroid associated orbitopathy," The Lancet, vol. 354, no. 9180, pp. 743-744, 1999.

[25] R. Villanueva, A. M. Inzerillo, Y. Tomer et al., "Limited genetic susceptibility to severe graves' ophthalmopathy: no role for CTLA-4 but evidence for an environmental etiology," Thyroid, vol. 10, no. 9, pp. 791-798, 2000.
[26] P. Alvarez-VAzquez, L. Constenla, R. V. Garcia-Mayor, A. Larranaga, and D. Valverde, "Association of CTLA4 gene polymorphism with ophthalmopathy of graves' disease in a Spanish population," International Journal of Endocrinology and Metabolism, vol. 9, no. 3, pp. 397-402, 2011.

[27] G. Borodic, D. M. Hinkle, and Y. Cia, "Drug-induced graves disease from CTLA-4 receptor suppression," Ophthalmic Plastic and Reconstructive Surgery, vol. 27, no. 4, pp. e87-e88, 2011.

[28] D. Saverino, R. Brizzolara, R. Simone et al., "Soluble CTLA4 in autoimmune thyroid diseases: relationship with clinical status and possible role in the immune response dysregulation," Clinical Immunology, vol. 123, no. 2, pp. 190-198, 2007.

[29] G. J. Freeman, V. A. Boussiotis, A. Anumanthan et al., "B7-1 and B7-2 do not deliver identical costimulatory signals, since B7-2 but not B7-1 preferentially costimulates the initial production of IL-4," Immunity, vol. 2, no. 5, pp. 523-532, 1995.

[30] L. J. Sigal, H. Reiser, and K. L. Rock, “The role of B7-1 and B72 costimulation for the generation of CTL responses in vivo," Journal of Immunology, vol. 161, no. 6, pp. 2740-2745, 1998.

[31] T. J. Smith, D. Sciaky, R. P. Phipps, and T. A. Jennings, “CD40 expression in human thyroid tissue: evidence for involvement of multiple cell types in autoimmune and neoplastic diseases," Thyroid, vol. 9, no. 8, pp. 749-735, 1999.

[32] F. Ye, P. Hou, X. Wu et al., "The significance of immune-related molecule expression profiles in an animal model of Graves' disease," Autoimmunity, vol. 45, no. 2, pp. 143-152, 2012.

[33] Y. Tomer, E. Concepcion, and D. A. Greenberg, "A C/T singlenucleotide polymorphism in the region of the CD40 gene is associated with Graves' disease," Thyroid, vol. 12, no. 12, pp. 1129-1135, 2002.

[34] E. M. Jacobson, E. Concepcion, T. Oashi, and Y. Tomer, "A graves' disease-associated Kozak sequence single-nucleotide polymorphism enhances the efficiency of CD40 gene translation: a case for translational pathophysiology," Endocrinology, vol. 146, no. 6, pp. 2684-2691, 2005.

[35] E. M. Jacobson, A. K. Huber, N. Akeno et al., "A CD40 Kozak sequence polymorphism and susceptibility to antibodymediated autoimmune conditions: the role of CD40 tissuespecific expression," Genes and Immunity, vol. 8, no. 3, pp. 205214, 2007.

[36] A. G. Gianoukakis and T. J. Smith, "Recent insights into the pathogenesis and management of thyroid-associated ophthalmopathy," Current Opinion in Endocrinology, Diabetes and Obesity, vol. 15, no. 5, pp. 446-452, 2008.

[37] J. Myśliwiec, D. Waligórski, A. Nikołajuk, and M. Górska, “Soluble CD40 and its ligand CD154 in patients with Graves' ophthalmopathy during combined therapy with corticosteroids and teleradiotherapy," Advances in Medical Sciences, vol. 52, pp. 104108, 2007.

[38] G. J. Kahaly, O. Shimony, Y. N. Gellman et al., "Regulatory T-cells in Graves' orbitopathy: baseline findings and immunomodulation by anti-T lymphocyte globulin," Journal of Clinical Endocrinology and Metabolism, vol. 96, no. 2, pp. 422429, 2011.

[39] D. Pan, Y.-H. Shin, G. Gopalakrishnan, J. Hennessey, and L. J. De Groot, "Regulatory T cells in Graves' disease," Clinical Endocrinology, vol. 71, no. 4, pp. 587-593, 2009. 


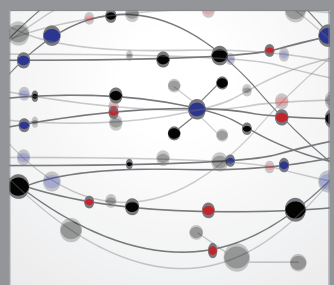

The Scientific World Journal
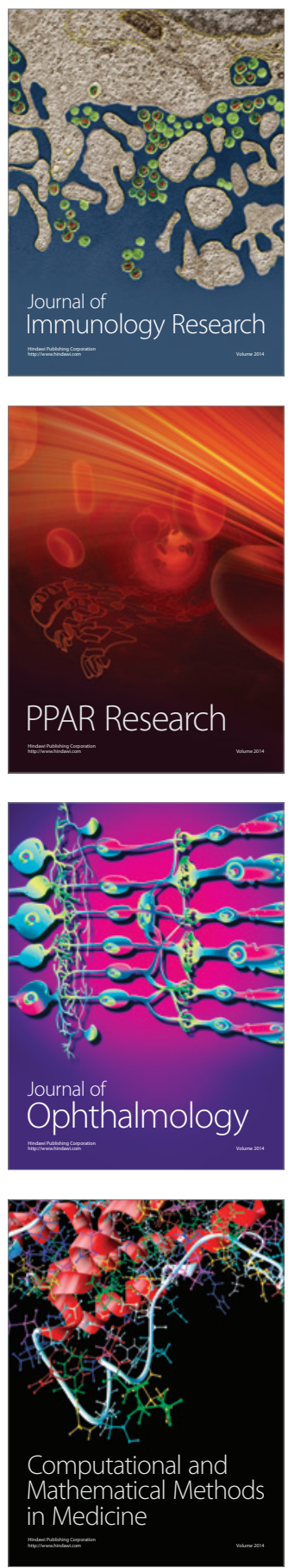

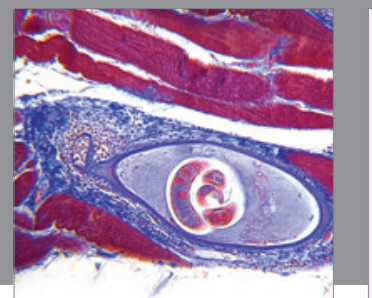

Gastroenterology

Research and Practice
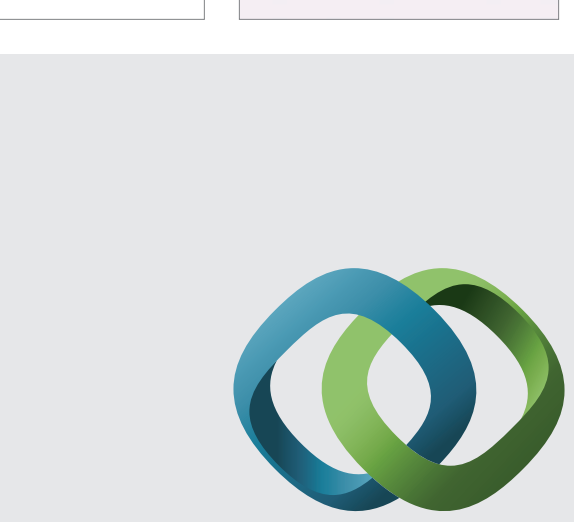

\section{Hindawi}

Submit your manuscripts at

http://www.hindawi.com
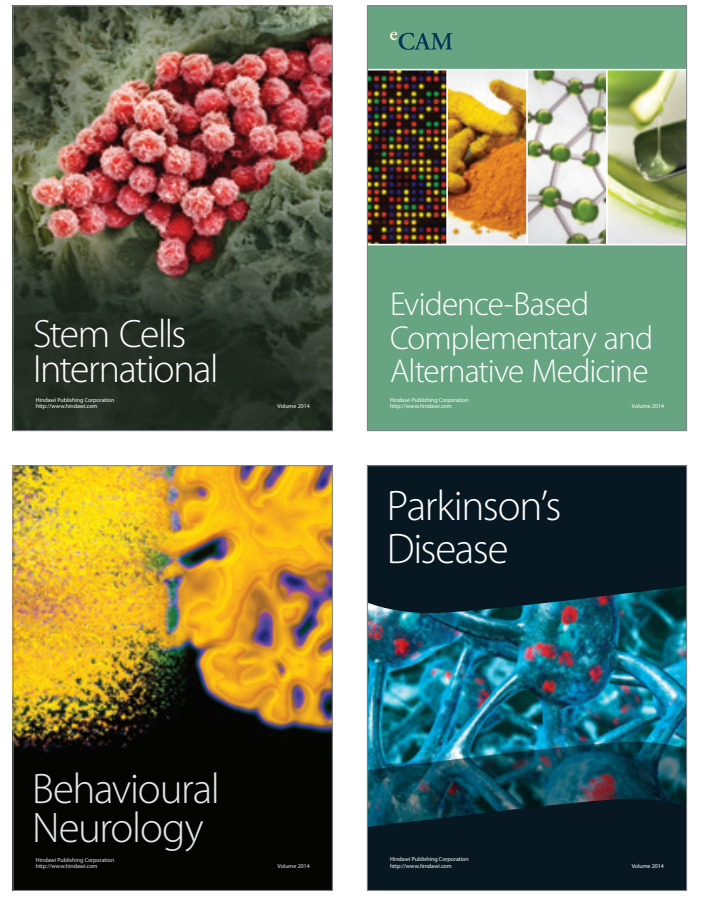
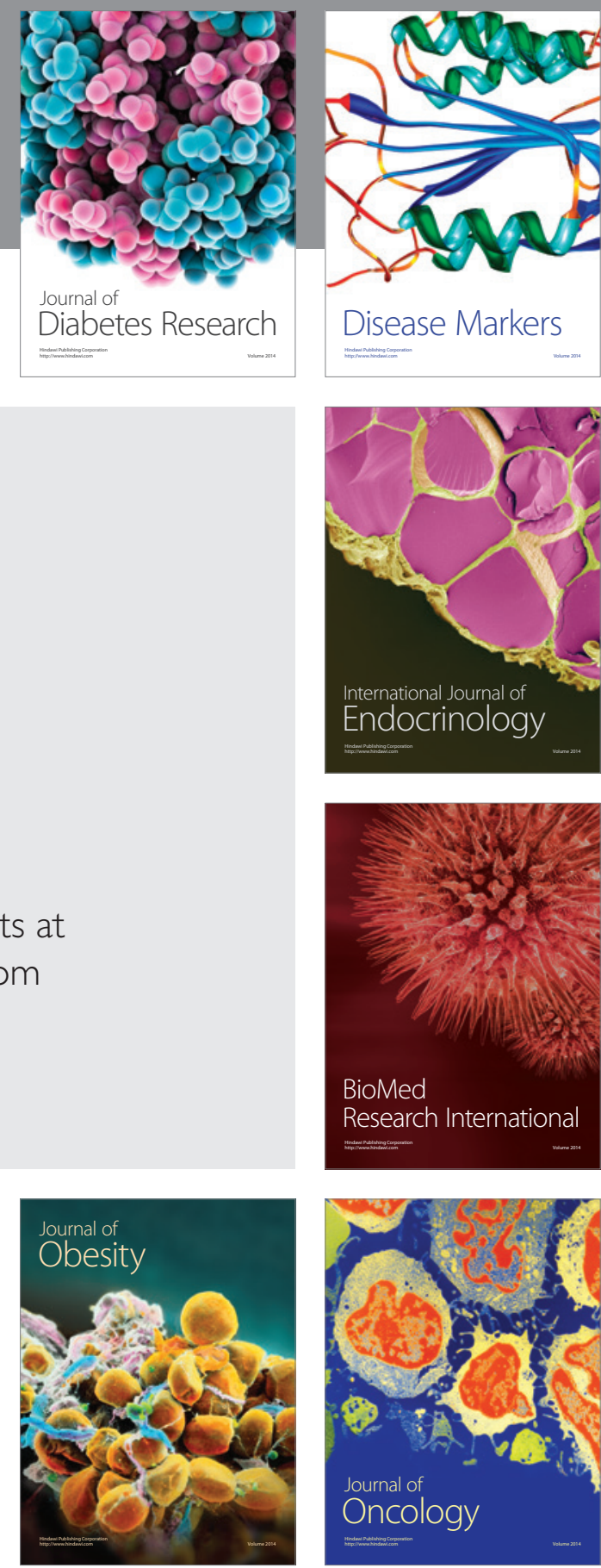

Disease Markers
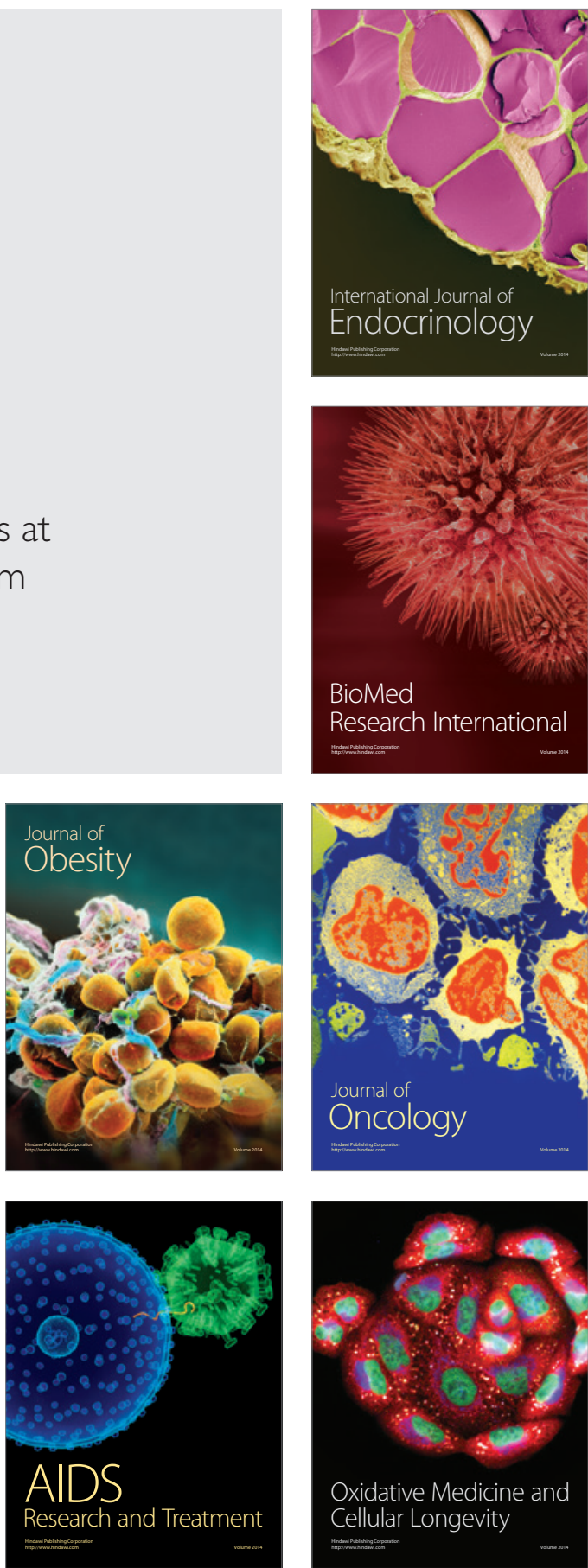